\title{
Nonthermal and thermal emission from the supernova remnant RX J1713.7-3946
}

\author{
E. G. Berezhko ${ }^{1}$ and H. J. Völk ${ }^{2}$ \\ 1 Yu.G. Shafer Institute of Cosmophysical Research and Aeronomy, 31 Lenin Ave., 677980 Yakutsk, Russia \\ e-mail: berezhko@ikfia.ysn.ru \\ 2 Max Planck Institut für Kernphysik, Postfach 103980, 69029 Heidelberg, Germany \\ e-mail: Heinrich.Voelk@mpi-hd.mpg.de
}

Received 17 September 2009 / Accepted 19 November 2009

\begin{abstract}
Aims. A nonlinear kinetic theory of cosmic ray (CR) acceleration in supernova remnants (SNRs) is employed to investigate the properties of SNR RX J1713.7-3946.

Methods. Observations of the nonthermal radio and X-ray emission spectra, as well as the HESS measurements of the very high energy $\gamma$-ray emission, are used to constrain the astronomical and CR acceleration parameters of the system. It is argued that RX J1713.73946 is a core collapse supernova (SN) of type II/Ib with a massive progenitor, and it has an age of $\approx 1600 \mathrm{yr}$ and is at a distance of $\approx 1 \mathrm{kpc}$. It is also assumed that the CR injection/acceleration takes place uniformly across the shock surface for this kind of core collapse SNR.

Results. The theory gives a consistent description for all the existing observational data, including the nondetection of thermal X-rays and the spatial correlation of the X-ray and $\gamma$-ray emission in the remnant. Specifically, it is shown that an efficient production of nuclear CRs, leading to strong shock modification and a strong downstream magnetic field $B_{\mathrm{d}} \approx 140 \mu \mathrm{G}$ can reproduce in detail the observed synchrotron emission from radio to X-ray frequencies, together with the $\gamma$-ray spectral characteristics as observed by the HESS telescopes.

Conclusions. The calculations are consistent with RX J1713.7-3946 being an efficient source of nuclear CRs.
\end{abstract}

Key words. ISM: cosmic rays - acceleration of particles - shock waves - ISM: individual objects: RX J1713.7-3946 radiation mechanisms: non-thermal - gamma rays: ISM

\section{Introduction}

The shell-type supernova remnant (SNR), RX J1713-3946, located in the Galactic plane was discovered in X-rays with ROSAT (Pfeffermann \& Aschenbach 1996). Subsequent studies of this SNR with the ASCA satellite by Koyama et al. (1997) and Slane et al. (1999), and later with XMM by Cassam-Chenaï et al. (2004), have not positively identified any thermal X-ray emission so they tentatively concluded that the observable X-ray emission is entirely nonthermal.

The radio emission, on the other, hand is quite weak. Only part of the remnant shell could be detected in radio synchrotron emission up to now, with a poorly known spectral form (Lazendic et al. 2004). For the spatially integrated radio flux, Aharonian et al. (2006) estimate about twice the value found by Lazendic et al. (2004). Most recently, Acero et al. (2009) have derived a total flux value of $22 \mathrm{Jy}<S<26 \mathrm{Jy}$ at $1.4 \mathrm{GHz}$, still about two times higher than estimated by Aharonian et al. (2006). As far as the estimate of the magnetic field strength is concerned, this higher estimate of the radio synchrotron flux increases the magnetic field estimate (see below).

RX J1713-3946 was also detected in very high-energy (VHE: >100 GeV) $\gamma$-rays with the CANGAROO (Muraishi et al. 2000; Enomoto et al. 2002) and HESS telescopes (Aharonian et al. 2004, 2006, 2007). Especially the latter, very detailed observations show a clear shell structure at $\mathrm{TeV}$ energies that correlates well with the ASCA contours.
In a first theoretical paper on this source (Berezhko \& Völk 2006), we investigated the acceleration of CR electrons and protons in detail, using nonlinear kinetic theory (Berezhko et al. 1996; Berezhko \& Völk 2000). Observations of the nonthermal radio and X-ray emission spectra, as well as the HESS measurements of the very high-energy $\gamma$-ray emission, were used to constrain the astronomical and the particle acceleration parameters of the system. Under the assumption that RX J1713.7-3946 is the remnant of a core collapse supernova ( $\mathrm{SN}$ ) of type II/Ib with a massive progenitor, the theory indeed gives a consistent description for the existing data on the nonthermal emission. The VHE data from HESS were specifically shown to be best explained as hadronic $\gamma$-ray emission, where the magnetic field amplification strongly depresses the inverse Compton and Bremsstrahlung fluxes. Subsequently (Berezhko \& Völk 2008), we analyzed the spatial correlation between the synchrotron and the VHE emission, which has been used before as an argument for a leptonic origin of the VHE emission (Katz \& Waxman 2008; Plaga 2008). It has been argued that correlated density and magnetic field strength variations lead rather naturally to a spatial correlation of the hadronic VHE emission with the synchrotron emission. Similar arguments have been brought forward by Tanaka et al. (2008). Also, the recent broad-band X-ray synchrotron measurements (Uchiyama et al. 2007; Takahashi et al. 2008) with the Suzaku instrument were compared to the theoretical synchrotron spectra and were found to be quite consistent with a hadronic model. A purely leptonic model, on the other hand 
(e.g. Porter et al. 2006), where magnetic field amplification is not expected, was shown to be inconsistent with these observations. Acero et al. (2009) have also considered a leptonic origin of the $\gamma$-ray emission as a viable possibility. This has to be compared with the results of the present paper.

These theoretical considerations assumed that only over part of the shock surface injection of suprathermal nuclear ions occurs effectively, because the magnetic field is essentially parallel to the shock surface over other parts. In fairly regular SNRs like those of type Ia explosions into a presumably uniform circumstellar environment, this is a significant effect, as in the case of SN 1006. For a blast wave propagating into the highly turbulent shell of a stellar wind bubble from a massive progenitor star, on the other hand, the magnetic flux tubes in the shell are presumably so slender and irregular that energetic particles can cross diffusively into regions where suprathermal injection is depressed, yet acceleration is possible. As a result, shock modification by the pressure gradient of the accelerating particles becomes possible everywhere on the shock surface. This reasoning follows the discussion in Berezhko et al. (2009) with the conclusion that SNRs propagating into wind bubbles should have their forward shock modified everywhere. Technically, this implies that the SNR becomes spherically symmetric and the correction factor $f_{\text {re }}<1$ (Völk et al. 2003) on the spherically symmetric solution reaches unity. The ubiquitous shock modification also has implications for the thermal emission, because the thermal gas, heated within the precursor due to the MHD wave damping and later in the subshock, only reaches lower temperatures than in the case of an unmodified shock.

In contrast to the previous studies (Berezhko \& Völk 2006, 2008) of RX J1713.7-3946, we therefore adopt here the approximation of spherically symmetric CR injection/acceleration with $f_{\text {re }} \approx 1$ (see also Zirakashvili 2009). It is demonstrated that in this approximation a consistent solution can also be found. In addition, a rough estimate of the thermal X-ray emission from this object is given. Even though the error in this estimate might be quite large, the nominal result is consistent with the present nondetection of thermal X-rays (e.g. Acero et al. 2009). Finally, the observed correlation of the X-ray and the VHE $\gamma$-ray emission is discussed in a generalized form.

\section{Results}

As in the previous studies (Berezhko \& Völk 2006, 2008), a source distance of $d=1 \mathrm{kpc}$ is adopted here. For the present angular source size of about 60 arcmin, this implies an SNR radius $R_{\mathrm{s}} \approx 10 \mathrm{pc}$. For the SNR age the value $t_{\mathrm{sn}}=1612 \mathrm{yr}$ is used, consistent with the hypothesis of Wang et al. (1997) that RX J1713-3946 is the remnant of the AD393 "guest star".

It had been argued (Berezhko \& Völk 2006) that the existing data are only consistent with RX J1713.7-3946 being a core collapse SN of type II/Ib. Those progenitor stars of core collapse $\mathrm{SNe}$ that emit intense winds are massive main-sequence stars with initial masses $M_{\mathrm{i}} \gtrsim 15 M_{\odot}$. During their evolution in a surrounding uniform ISM of gas number density $\varrho_{0}=m_{\mathrm{p}} N_{\text {ISM }}$, the wind termination shock creates a hot bubble of shocked wind material, surrounded by a turbulent shell of shocked ISM behind an outer forward shock that communicates the internal overpressure to the environment.

The gas number density distribution $N_{\mathrm{g}}(r)=\varrho(r) / m_{\mathrm{p}}$ is assumed to have the form

$N_{\mathrm{g}}=0.008+0.24[\mathrm{r} /(10 \mathrm{pc})]^{12} \mathrm{~cm}^{-3}$,

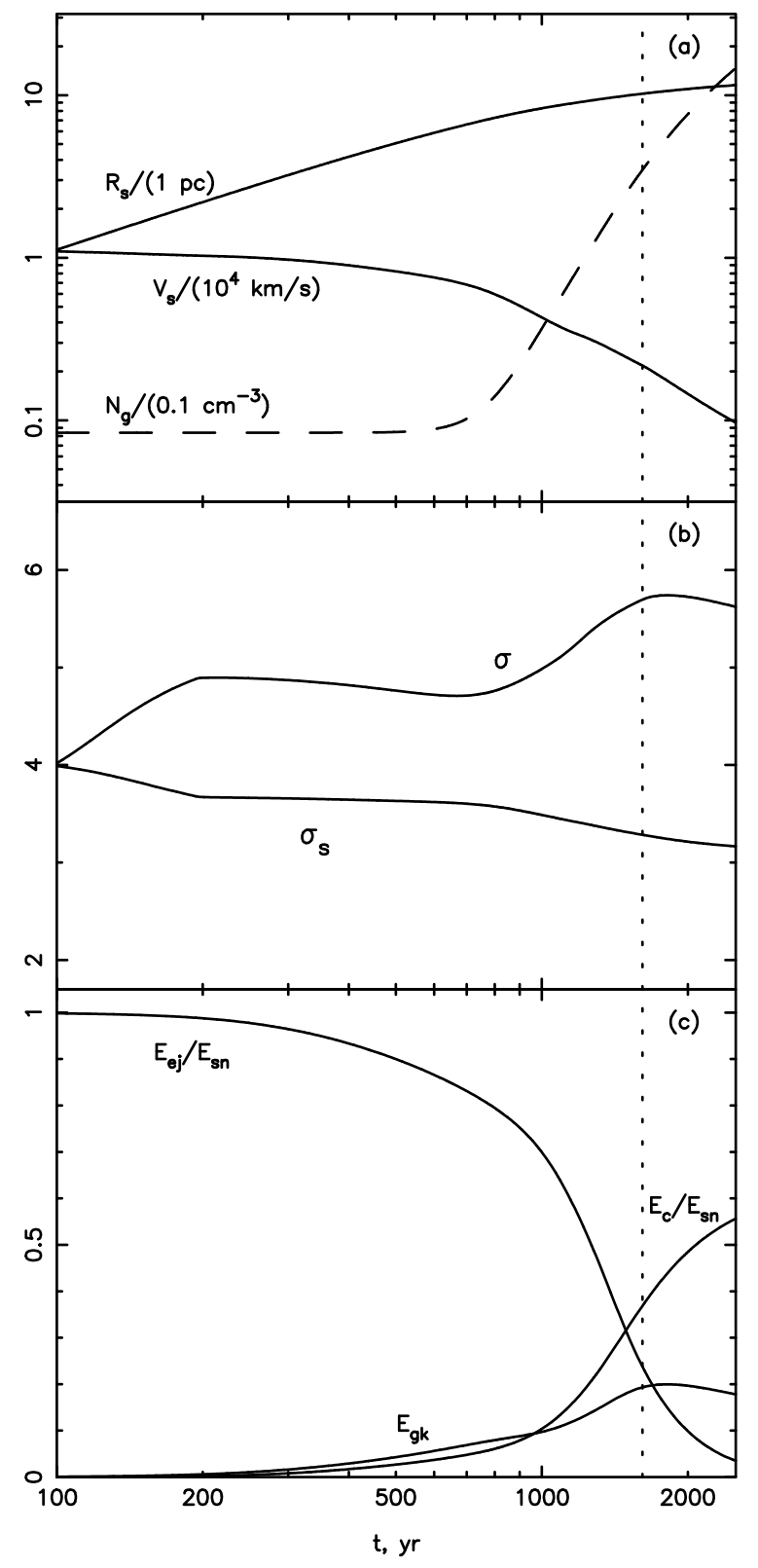

Fig. 1. a) Shock radius $R_{\mathrm{s}}$, shock speed $V_{\mathrm{s}}$, and preshock gas density $N_{\mathrm{g}}$; b) total shock $(\sigma)$ and subshock $\left(\sigma_{\mathrm{s}}\right)$ compression ratios; c) ejecta $\left(E_{\mathrm{ej}}\right)$ gas-kinetic $\left(E_{\mathrm{gk}}\right)$ and $\mathrm{CR}\left(E_{\mathrm{c}}\right)$ energies as a function of time, normalized to the total mechanical SNR energy $E_{\mathrm{sn}}$. The vertical dotted line marks the current evolutionary epoch.

corresponding to a very low-density bubble $\left(N_{\mathrm{b}}=0.008 \mathrm{~cm}^{-3}\right)$ for $r \ll 10 \mathrm{pc}$, plus a power-law distribution of the shell density. This adopted analytical form consistently models the gas density distribution within the bubble, created by the wind of the progenitor star (Berezhko \& Völk 2006). The density profile corresponds to a gas number density $N_{\mathrm{g}}=0.25 \mathrm{~cm}^{-3}$ at the current $\mathrm{SN}$ shock position, which is a factor $\sim 5$ lower than in Berezhko \& Völk (2006).

Under these conditions an SN explosion energy $E_{\mathrm{sn}}=1.3 \times$ $10^{51} \mathrm{erg}$ and an ejecta mass $M_{\mathrm{ej}}=3 M_{\odot}$ lead to a good fit for the observed SNR properties. To determine the explosion energy directly from the data, the value of the shock speed $V_{\mathrm{s}}$ would have been needed. However, this quantity is not known. The ejecta energy $E_{\text {ej }}$ is still about $0.25 E_{\mathrm{sn}}$. Together with the kinetic energy of the shocked gas $E_{\mathrm{gk}} \approx 0.2 E_{\mathrm{sn}}$, this makes up about half of the total energy $E_{\text {sn }}$ (see Fig. 1). 
The spectral fit, also for the $\gamma$-ray data, can be achieved with the values $\eta=5 \times 10^{-4}$ of the proton injection rate and $B_{\mathrm{d}}=142 \mu \mathrm{G}$ for the downstream magnetic field strength. Such values of $B_{\mathrm{d}}$ are significantly higher than for typical ambient magnetic fields, also for the considered bubble wall in the dense environment. (The gas density at the shock is still a factor $\sim 100$ lower than in that environment, which implies a strongly decompressed field upstream of the SNR shock compared to the value in the ambient medium.) The high $B_{\mathrm{d}}$-value must be attributed to field amplification at the shock front because of the strong wave production by the acceleration of CRs far into the nonlinear regime (Lucek \& Bell 2000; Bell 2004) ${ }^{1}$.

It is assumed that also electrons are injected into the acceleration process at the shock front. Since the details of the electron injection process are poorly known, the electron injection rate is chosen such that the electron to proton ratio $K_{\text {ep }}$ (defined as the ratio of their distribution functions at all rigidities where the protons are already relativistic and the electrons have not yet been cooled radiatively) is a constant to be determined from the synchrotron observations. Clearly, from the point of view of injection/acceleration theory, $K_{\mathrm{ep}}$, together with $B_{0} \equiv B_{\mathrm{d}} / \sigma$ and $\eta$, must be treated as theoretically not very well-constrained parameters to be quantitatively determined by comparison with the available synchrotron and $\gamma$-ray data. Here, $\sigma$ is the overall shock compression ratio.

In the present case, the following parameter values were obtained by iteration: $\eta=5 \times 10^{-4}, B_{0}=25 \mu \mathrm{G}, K_{\text {ep }}=1.3 \times 10^{-3}$. The gas dynamic variables at the present epoch are $\sigma=5.7$, $\sigma_{\mathrm{s}}=3.3$, and $V_{\mathrm{s}} \approx 2200 \mathrm{~km} \mathrm{~s}^{-1}$, where $\sigma_{\mathrm{s}}$ and $V_{\mathrm{s}}$ denote the subshock compression ratio and the overall shock velocity, respectively (Fig. 1).

The corresponding solutions of the dynamic equations at each instant of time yield the CR spectrum and the spatial distributions of CRs and thermal gas, hence also the expected fluxes of nonthermal emission produced by the accelerated CRs. The overall broadband spectral energy distribution (SED), expected at the current evolutionary epoch, is displayed in Fig. 2, together with the experimental data from ATCA at radio wavelengths, as estimated for the full remnant by Acero et al. (2009), the X-ray data from ASCA (Aharonian et al. 2006) and Suzaku (Uchiyama et al. 2007), and the HESS $\gamma$-ray data (Aharonian et al. 2007).

The comparison with our earlier theoretical spectrum (Berezhko \& Völk 2008) shows that also a model with full spherical symmetry can successfully describe the spatially integrated nonthermal emission properties of this object.

At energies $\epsilon_{\gamma}<1 \mathrm{TeV}$ the theoretical $\gamma$-ray spectrum is as hard as $\mathrm{d} F_{\gamma} / \mathrm{d} \epsilon_{\gamma} \propto \epsilon_{\gamma}^{-1.8}$, whereas for $\epsilon_{\gamma}>10 \mathrm{TeV}$ it has a smooth cutoff. It should be noted that the $\gamma$-ray cutoff energy $\epsilon_{\gamma}^{\max } \approx 0.1 c p_{\max }$ is sensitive to the magnetic field strength $B_{\mathrm{d}}$, since the proton cutoff momentum has a dependence $p_{\max } \propto$ $R_{\mathrm{S}} V_{\mathrm{s}} B_{\mathrm{d}}$ (Berezhko 1996). Note that the energy loss time of energetic protons due to their hadronic collisions with the background nuclei is so long $\sim 10^{8} \mathrm{yr}$, that it does not play any role in determining $p_{\max }$.

The theoretical results presented here are fully consistent with a dominantly hadronic origin of the observed $\mathrm{TeV} \gamma$-rays . At the present epoch, the SNR has already converted $\approx 35 \%$ of $E_{\text {sn }}$ into accelerated nuclei.

\footnotetext{
${ }^{1}$ For direct observational evidence regarding magnetic field amplification in RX J1713.7-3946, see Völk et al. (2005); Ballet (2006); Berezhko \& Völk (2006).
}

\subsection{Estimate of the thermal X-ray emission}

As for the case of RX J0852.0-4622 (Berezhko et al. 2009) in addition the expected flux of thermal X-rays is estimated here. This is only possible in a very approximate way, even if we disregard the ejecta emission with the argument that the ejected mass in the present phase is small compared to the swept-up mass. The reason is the explosion into the wind bubble, creating a rather different thermodynamical structure from that of a classical Sedov solution for an SN explosion into a uniform medium, until now exclusively considered in the literature. Nevertheless the remnant of RX J1713.7-3946 in the stellar wind shell is approaching a roughly self-similar evolutionary phase, modified by strong particle acceleration relative to a purely gas dynamic evolution. Other estimates of the thermal emission from RX J1713.7-3946 have been made by Katz \& Waxman (2008) and Drury et al. (2008), effectively assuming an explosion into a uniform medium.

The approximation adopted here is the following. The present bubble case is compared with an SNR in a uniform medium in the classical Sedov phase without any CR acceleration, making four assumptions: (i) the total hydrodynamic explosion energy is the same in both cases; (ii) the shock velocity is the same; (iii) the present gas density upstream of the shock is the same; and (iv) the two objects are at the same distance of $1 \mathrm{kpc}$. Then the results of Hamilton et al. (1983) for the thermal X-ray flux from a classical Sedov SNR are used, employing the emission measure of the bubble remnant instead of that of the classical Sedov remnant with the same four parameters above. The thermal X-ray emission at $\mathrm{keV}$-energies is dominated by lines, so it cannot be approximated by some simple analytical formula. We use the results of numerical calculations performed by Hamilton et al. (1983) with some corrections. These mean that the X-ray emissivity of the remnant is reduced by the ratio $R_{\mathrm{em}}=E M_{\mathrm{b}} / E M_{\mathrm{S}}$ of the emission measure $E M_{\mathrm{b}}$ for our bubble solution to the emission measure for the classical Sedov solution $E M_{\mathrm{S}}$, which corresponds to a uniform ambient gas density $N_{\mathrm{g}}\left(R_{\mathrm{s}}\right)$ (Berezhko et al. 2009).

Also the relation

$\frac{T_{\mathrm{sub}}}{T_{\mathrm{s}}}=\frac{\left[\left(\sigma_{\mathrm{s}}-1\right)(\gamma+1)+2\right](\gamma+1)^{2}}{4 \gamma(\gamma-1) \sigma^{2}}$

is used, with $\gamma=5 / 3$, to determine the subshock temperature $T_{\text {sub }}$, relevant for the thermal emission flux for the modified shock. The quantity $T_{\mathrm{s}}=10^{7}\left(V_{\mathrm{s}} /\left(839 \mathrm{~km} \mathrm{~s}^{-1}\right)\right)^{2} \mathrm{~K} \approx 6.9 \times 10^{7} \mathrm{~K}$ denotes the gas temperature of an unmodified shock with $V_{\mathrm{s}} \approx$ $2200 \mathrm{~km} \mathrm{~s}^{-1}$.

Using the differential thermal X-ray model spectra $\mathrm{d} F / \mathrm{d} \epsilon \approx$ $3 \times 10^{-5}$ photons/( $\mathrm{keV} \mathrm{cm}^{2} \mathrm{~s}$ ) from Hamilton et al. (1983) (for $T_{\mathrm{e}} \neq T_{\mathrm{i}}$, see their Fig. 2) for their $\eta=N_{\mathrm{H}}^{2} E_{\mathrm{sn}}=10^{49} \mathrm{erg} \mathrm{cm}^{-6}$ taking into account the scaling $\mathrm{d} F / \mathrm{d} \epsilon \propto \eta$ with our model numbers $\eta \approx 8 \times 10^{49} \mathrm{erg} \mathrm{cm}^{-6}$, multiplying it by the factor $\theta^{2}\left[E_{\mathrm{sn}} /\left(10^{51} \mathrm{erg}\right)\right]^{-1 / 2}$, as required, where $\theta=54.5$ arcmin for the angular size of the classical Sedov remnant corresponding to RX J1713.7-3946, and multiplying it also by the factor $R_{\mathrm{em}}=0.46$, results in a thermal spectral energy density $\epsilon^{2} \mathrm{~d} F / \mathrm{d} \epsilon \approx 288 \mathrm{eV} \mathrm{cm}^{-2} \mathrm{~s}^{-1}$ for $\epsilon=1 \mathrm{keV}$.

This result has not yet taken into account that the actual postshock gas temperature $T_{\text {sub }}$ is lower by a factor $\approx 0.41$ than the temperature $T_{\mathrm{s}}$ used in the above estimate. As discussed in Berezhko et al. (2009), this roughly corresponds to a reduced parameter value $\eta \approx 3.3 \times 10^{49} \mathrm{erg} \mathrm{cm}^{-6}$ and thus to a reduction in the thermal flux at $1 \mathrm{keV}$ to $\epsilon^{2} \mathrm{~d} F / \mathrm{d} \epsilon \approx 118 \mathrm{eV} \mathrm{cm}^{-2} \mathrm{~s}^{-1}$. 


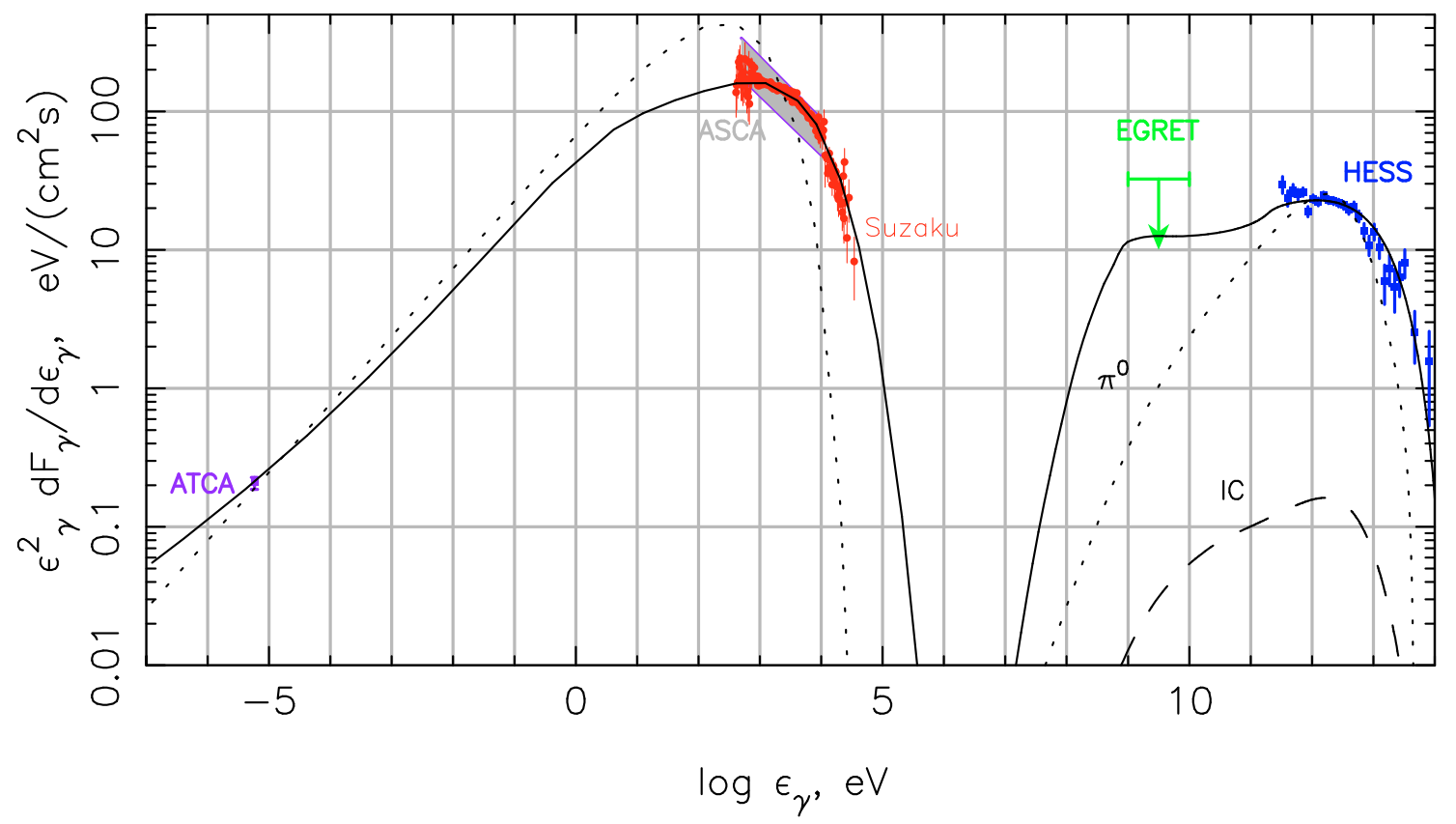

Fig. 2. Spatially integrated, overall nonthermal spectral energy distribution of RX J1713.7-3946. The solid curve at energies above $10^{7} \mathrm{eV}$ corresponds to $\pi^{0}$-decay $\gamma$-ray emission, whereas the dashed curve indicates the Inverse Compton (IC) emission. The dotted line corresponds to the test particle limit which implies insignificant proton acceleration and magnetic field amplification (see Berezhko \& Völk 2008, for details). The ATCA radio data, as derived by Acero et al. (2009), the ASCA X-ray data (cf. Aharonian et al. 2006), the Suzaku X-ray data (Uchiyama et al. 2007), and the 2006 HESS $\gamma$-ray data (Aharonian et al. 2007) are also shown. The EGRET upper limit for the RX J1713.7-3946 position (Aharonian et al. 2006) is included as well.

These numbers must be compared with the observed nonthermal X-ray energy flux $\epsilon^{2} \mathrm{~d} F / \mathrm{d} \epsilon \approx 200 \mathrm{eV} \mathrm{cm}^{-2} \mathrm{~s}^{-1}$ at $1 \mathrm{keV}$ (see Fig. 2). Therefore, at $1 \mathrm{keV}$, the thermal energy flux is smaller than the nonthermal flux by a factor of about 2 . This does not contradict the nondetection of thermal X-rays from RX J1713.7-3946.

\subsection{Spatial correlation of the nonthermal $X$-ray fluxes with the $\gamma$-ray fluxes}

Acero et al. (2009) find a positive spatial nonlinear correlation $F_{\mathrm{X}} \propto F_{\gamma}^{\alpha}$ with $\alpha=2.41$ between the flux $F_{\gamma}$ of $\gamma$-rays with energies $\epsilon_{\gamma}=1-10 \mathrm{TeV}$ and the flux of nonthermal X-rays $F_{\mathrm{X}}$ with energies $\epsilon_{\gamma}=1-10 \mathrm{keV}$, and conclude that such a correlation favors a leptonic nature of the $\mathrm{TeV}$-emission. Such a positive correlation is naturally expected within the present approach.

\subsubsection{Correlation of the radial profiles}

In terms of the spherically symmetric model, discussed here, the correlation between X-ray and $\gamma$-ray fluxes means that the projected radial profiles $J_{\mathrm{X}}(\rho)$ and $J_{\gamma}(\rho)$ of these emissions as functions of projected radial distance $\rho$ have similar shapes. At first sight this contradicts this model: as can be seen from Figs. 3 and 4, the calculated profiles are significantly different. Both profiles have a sharp peak just behind the shock front, but the X-ray profile is by a factor of about 3 thinner than the $\gamma$-ray profile. However, when smoothed with the point spread function of width $\sigma_{0}=0.05^{\circ}$, corresponding to the HESS angular resolution, these profiles become very similar. Acero et al. (2009) compared XMM measurements of the X-ray emission smoothed with a point spread function of width $\sigma=0.05^{\circ}$. It can therefore be concluded that the similarity of the X-ray and the $\gamma$-ray radial profiles is a trivial consequence of smoothing two differently sharp peaks by a point spread function whose width is considerably greater than the widths of either one of the emission peaks.

The experimentally observed correlation according to Fig. 9 of Acero et al. (2009) is fairly close to the relation $F_{\mathrm{X}}=F_{\gamma}$ for all the brighter SNR regions. Some deviation from this relation is observed for six of the outer and dimmer regions, for which $F_{\mathrm{X}}<F_{\gamma}$. This peculiarity can also be explained within the present model. The reason is that protons with an energy of about $100 \mathrm{TeV}$ occupy a region, that is noticeably larger than the shock size $R_{\mathrm{s}}$, because of their high diffusive mobility. The $10 \mathrm{TeV} \gamma$-ray emission these protons produce has an even larger radial extension since the gas density strongly increases with radial distance. Such an effect is absent for the electrons of the same energy which produce the nonthermal keV-emission. As a result of their strong energy losses, they occupy a region thinner than $10^{-2} R_{\mathrm{S}}$ around the shock front. Therefore, as can be clearly seen from Figs. 3 and 4 , the ratio $F_{\gamma} / F_{\mathrm{X}}$ is expected to increase as a function of radial distance for $\rho / R_{\mathrm{S}}>1$, as the data of Acero et al. (2009) are interpreted here.

\subsubsection{Correlation of azimuthal variations}

The measured X-ray and $\gamma$-ray emissions also undergo rather closely correlated azimuthal variations across the remnant. Such an effect cannot be described within the present model model, because it is spherically symmetric. One can nevertheless attempt to interpret the correlated behavior of the fluxes $F_{\mathrm{X}}$ and $F_{\gamma}$ in different azimuthal sectors of the remnant. It is natural to assume that this variation in the remnant properties stems from variations in the ambient gas density $\varrho$. According to the model proposed here, the SNR's present evolutionary phase is intermediate between the sweep-up and a quasi-Sedov phase. Since the ejecta kinetic energy, together with the kinetic energy of 


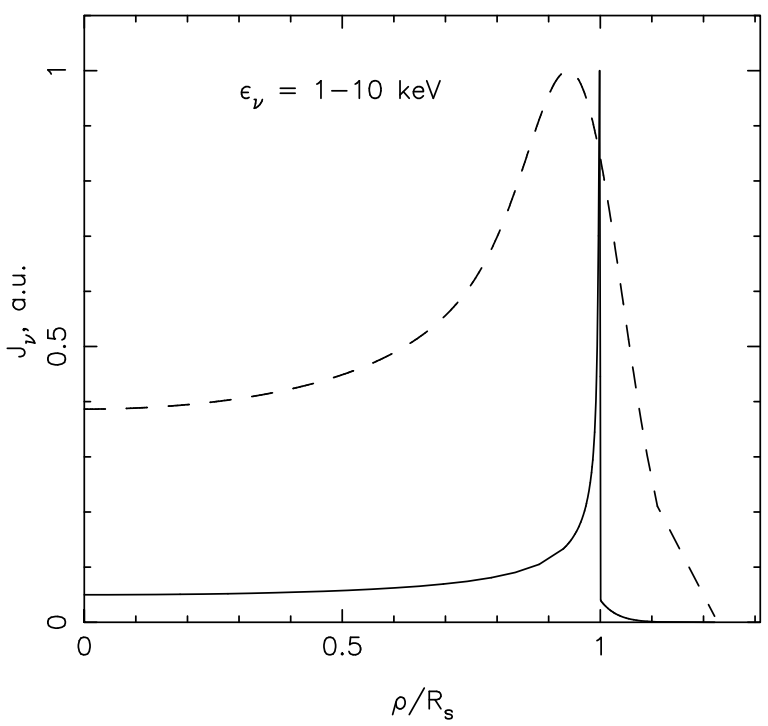

Fig. 3. The X-ray emissivities for the energy $\epsilon_{\gamma}=1-10 \mathrm{keV}$ as a function of projected, normalized radial distance $\rho / R_{\mathrm{s}}$. The calculated radial profile is represented by the solid line. The dashed line represents the calculated profile convolved with the point spread function of Gaussian width $0.05^{\circ}$.

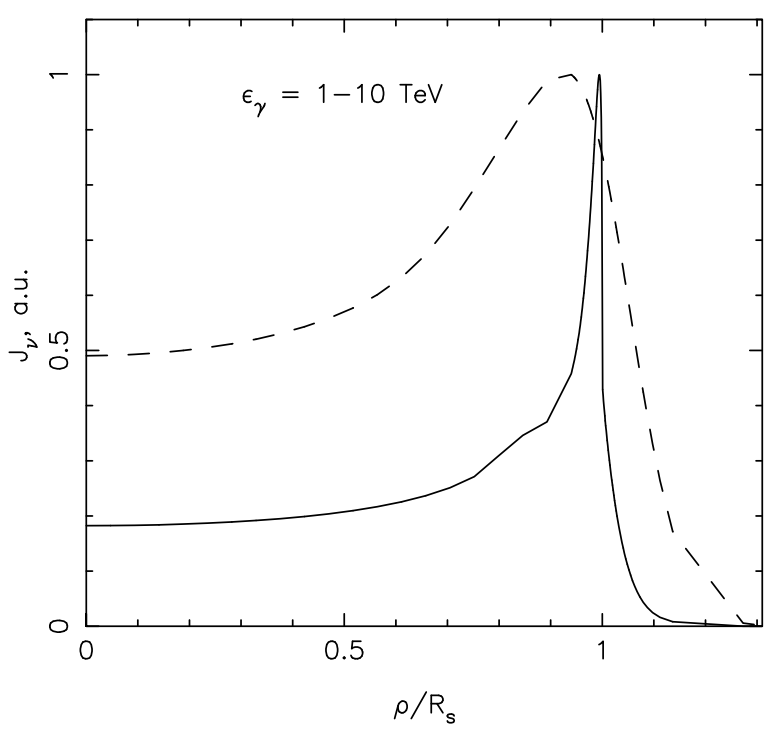

Fig. 4. The $\gamma$-ray emissivities for the $\gamma$-ray energy $\epsilon_{\gamma}=1-10 \mathrm{TeV}$ as a function of projected, normalized radial distance $\rho / R_{\mathrm{S}}$. The calculated radial profile is represented by the solid line. The dashed line represents the calculated profile convolved with the point spread function of Gaussian width $0.05^{\circ}$.

the swept-up gas, contains about half of the explosion energy, the SN shock can be approximately treated as a piston-driven shock rather than a pressure-driven shock in the Sedov phase. The speed of such a shock depends only weakly upon the upstream gas density. Therefore $V_{\mathrm{s}}$ can be approximated by a constant across the whole remnant.

The local hadronic $\gamma$-ray emission then varies like $F_{\gamma} \propto$ $\varrho E_{\mathrm{c}} \propto \varrho$, where the local CR energy content $E_{\mathrm{c}}$ in each angular sector is assumed to be the same everywhere, because the overall CR energy content $E_{\mathrm{c}} \approx 0.4 E_{\mathrm{SN}}$ has already reached the saturation level and therefore cannot undergo significant variations. In addition, the highest energy CRs in the SNR have the highest diffusive mobility so that they tend to be distributed roughly uniformly across the remnant.

As is clear from Fig. 2, the synchrotron spectrum at high energies $\epsilon_{\gamma}>10 \mathrm{eV}$ is already considerably influenced by synchrotron losses of the emitting electrons. Since the energy of these electrons is fairly rapidly and completely transformed into synchrotron emission, the flux $F_{\mathrm{X}} \propto K_{\mathrm{ep}} \varrho V_{\mathrm{s}}^{3}$ is determined by the available energy flux $\rho V_{\mathrm{s}}^{3} / 2$ into the shock, an essential part of which is transformed at the shock front into the energy flux of high-energy CRs. High-energy electrons therefore accumulate a fraction $K_{\text {ep }}$ of this flux. This flux hardly depends on the value of the interior magnetic field in all those SNR regions where the field is sufficiently high, $B_{\mathrm{d}} \approx 140 \mu \mathrm{G}$. In this case the relation $F_{\mathrm{X}} \propto F_{\gamma}$ is expected for the brightest part of remnant. This is indeed observed (Acero et al. 2009). Since the energy density of the amplified magnetic field $B_{\mathrm{d}}^{2}$ is phenomenologically known to be proportional to $\varrho V_{\mathrm{s}}^{\beta}$, with $2 \leq \beta \leq 3$ (Völk et al. 2005), it is expected to be proportional to the local upstream gas density $\varrho$. The magnetic field strength is therefore lower than the average value $\approx 140 \mu \mathrm{G}$ within those parts of the remnant where the gas density is lower than the average value. Synchrotron losses become much smaller in these regions and therefore the expected synchrotron X-ray flux is approximately $F_{\mathrm{X}} \propto \varrho K_{\mathrm{ep}} B_{\mathrm{d}}^{3 / 2} \propto \varrho^{7 / 4}$. For the dim part of the remnant, in which X-ray and $\gamma$-ray emission is considerably lower than on average, one then obtains $F_{\mathrm{X}} \propto F_{\gamma}^{7 / 4}$. This is approximately consistent with the observational result.

\section{Summary}

The assumption that $\mathrm{CR}$ injection/acceleration takes place uniformly across the whole SN shock surface is consistent with the existing data. The swept-up mass is so low in this case that, in a rough approximation, the estimated flux of thermal X-rays at $1 \mathrm{keV}$ is lower than the nonthermal flux, consistent with the nondetection of thermal X-ray emission until now.

It is concluded that the present observational knowledge of SNR RX J1713.7-3946 can be interpreted by a source that ultimately converts more than $35 \%$ of the mechanical explosion energy into nuclear CRs. Also, the observed high-energy $\gamma$-ray emission of SNR RX J1713.7-3946 turns out to primarily be of hadronic origin.

Acknowledgements. The authors thank V. S. Ptuskin and V. N. Zirakashvili for discussions of the thermal emission properties. This work was supported in part by the Russian Foundation for Basic Research (grants 06-02-96008, 0702-0221). E.G.B. acknowledges the hospitality of the Max-Planck-Institut für Kernphysik, where part of this work was carried out.

\section{References}

Abbott, D. C. 1982, ApJ, 263, 723

Acero, F., Ballet, J., Decourchelle, A., et al. 2009, A\&A, 505, 157

Aharonian, F. A., Akhperjanian, A., Aye, K.-M., et al. (HESS Collaboration) 2004, Nature, 432, 75

Aharonian, F. A., Akhperjanian, A., Bazer-Bachi, A. R., et al. (HESS Collaboration) 2006, A\&A, 449, 223

Aharonian, F. A., Akhperjanian, A., Bazer-Bachi, A. R., et al. (HESS Collaboration) 2007, A\&A, 464, 235

Ballet, J. 2006, Adv. Space Res., 37, 1902

Bell, A. R. 2004, MNRAS, 353, 550

Berezhko, E. G. 1996, Astropart. Phys., 5, 367

Berezhko, E. G. 2005, Adv. Space Res., 35, 1031

Berezhko, E. G. 2008, Adv. Space Res., 41, 429

Berezhko, E. G., \& Völk, H. J. 2000, A\&A, 357, 183

Berezhko, E. G., \& Völk, H. J. 2006, A\&A, 451,981

Berezhko, E. G., \& Völk, H. J. 2008, A\&A, 492,695 
A\&A 511, A34 (2010)

Berezhko, E. G., Elshin, V. K., \& Ksenofontov, L. T. 1996, JETPh, 82, 1 Berezhko, E. G., Pühlhofer, G., \& Völk, H. J. 2009, A\&A, 505, 641 Cassam-Chenaï, G., Decourshelle, A., Ballet, J., et al. 2004, A\&A, 427, 199 Drury, L.O'C., Aharonian, F. A., Malyshev, D., \& Gabici, S. 2008, A\&A, 496, 1 Enomoto, R., Tanimori, T., Naito, T., et al. 2002, Nature, 416, 823 Enomoto, R., Watanabe, S., Tanimori, T., et al. 2006, ApJ, 652, 1268 Fukui, Y., Moriguchi, Y., Tamura, K., et al. 2003, PASJ, 55, L61 Hamilton, A. J. S., Sarazin, C. L., \& Chevalier, R. A. 1983, ApJS, 41, 115 Hiraga, J. S., Uchiyama, Y., Takahashi, T., et al. 2005, A\&A, 431, 953 Katz, B., \& Waxman, E. 2008, JCAP, 01, 1

Koyama, K., Kinagasa K., Matsuzaki, K., et al. 1997, PASJ, 49, L7

Lazendic, J. S., Slane, P. O., Gaensler, B. M., et al. 2004, ApJ, 602, 271 Lucek, S. G., \& Bell, A. R. 2000, MNRAS, 314, 65

Moriguchi, Y., Tamura, T., Tawara, Y., et al. 2005, ApJ, 641, 947

Muraishi, H., Tanimori, T., \& Yanagita, S. 2000, A\&A, 354, L57
Pfeffermann, E., \& Aschenbach, B. 1996, in Röntgenstrahlung from the Universe, ed. H. U. Zimmermann, J. Trümper, \& H. Yorke, MPE Rep., 263, Garching, 267

Plaga, R. 2008, New Astron., 13, 73

Porter, T. A., Moskalenko, I. V., \& Strong, A. W. 2006, ApJ, 648, L29 Slane, P., Gaensler, B. M., Dame, T., et al. 1999, ApJ, 357, SL99

Takahashi, T., Tanaka, T., Uchiyama, Y., et al. 2008, PASJ, 60, S131 Tanaka, T., Uchiyama, Y., Aharonian, F. A., et al. 2008, ApJ, 685, 988 Uchiyama, Y., Aharonian, F. A., Tanaka, T., et al. 2007, Nature, 449, 576 Völk, H. J., Berezhko, E. G., \& Ksenofontov, L. T. 2003, A\&A, 409, 563 Völk, H. J., Berezhko, E. G., \& Ksenofontov, L. T. 2005, A\&A, 433, 229 Wang, Z. R., Qu, Q.-Y., \& Chen, Y. 1997, A\&A, 318, L59

Zirakashvili, V. N. 2009, in High Energy Gamma-Ray Astronomy, ed. F. A. Aharonian, W. Hofmann, \& F. M. Rieger, Melville, New York, AIP Conf. Proc., 1085, 129 